\title{
OPEN Development of surface engineered antigenic exosomes as vaccines for respiratory syncytial virus
}

\author{
Suyeon Hong ${ }^{1}$, Shaobo Ruan ${ }^{2}$, Zachary Greenberg $\mathbb{1}^{2}$, Mei He${ }^{2}$ \& Jodi L. McGill1ه
}

Respiratory syncytial virus (RSV) is one of the main pathogens associated with lower respiratory tract infections in infants and young children worldwide. Exosomes secreted by antigen presenting cells (APCs) can elicit immune responses by carrying major histocompatibility complex (MHC) class I molecules complexed with antigenic peptides and other co-stimulating factors. Therefore, we developed novel immunomagnetic nanographene particles to sequentially isolate, surface engineer, and release intact dendritic cell (DC) exosomes for use as a potential vaccine platform against RSV. The $\mathrm{H}-2 \mathrm{D}^{\mathrm{b}}$-restricted, immunodominant peptides from RSV $\left(\mathrm{M}_{187-195}\right.$ and $\left.\mathrm{NS1}_{61-75}\right)$ were introduced to MHC-I on DC-derived exosomes to express peptide/MHC-I (PMHC-I) complexes. A mouse model of RSV infection was used to define the immunogenicity of surface engineered exosomes for activating virusspecific immune responses. Ex vivo assays demonstrated that engineered exosomes carrying RSVspecific peptides can elicit interferon-gamma (IFN- $\gamma$ ) production by virus-specific CD8+ T cells isolated from RSV-infected C57BL/6 mice. In vivo assays demonstrated that subcutaneous administration of both $\mathrm{M}_{187-195}$ and $\mathrm{NS1}_{61-75}$ engineered exosomes to mice, with or without additional adjuvant, appeared safe and well tolerated, however, did not prime antigen-specific CD8+T cell responses. Surface engineered exosomes are immunogenic and promising for further development as a vaccine platform.

Respiratory syncytial virus (RSV) is one of the main causes of severe lower respiratory tract infections in infants and young children. The incidence of RSV-associated acute lower respiratory infection has been estimated approximately 33.1 million in 2015 globally, with total deaths more than 118,000 in children younger than age $5^{1}$. Despite years of research, there are currently no available licensed vaccines against human RSV infection. Hence, it is important to develop effective vaccines to prevent such viral infections and control RSV-induced disease.

Exosomes are small (30-150 nm) extracellular vesicles (EVs) secreted from living cells and involved in intercellular communication by containing various signaling molecules such as lipids, proteins, DNAs, and $\mathrm{RNAs}^{2,3}$. Several studies have reported that exosomes secreted from immune cells are immunogenic and can transport molecules over long distances for eliciting immune responses ${ }^{4}$. For example, exosomes released by B cells carry major histocompatibility complex (MHC) class II on their surface and have the capacity to stimulate $\mathrm{T}$ cells $s^{5}$. Human natural killer (NK) cell-derived exosomes expressing NK cell markers exhibit cytotoxicity against tumor cells ${ }^{6}$. Exosomes from Mycobacterium bovis Bacillus Calmette-Guérin (BCG)-infected macrophages also have the capacity to stimulate CD4+ and CD8+ T cells from BCG-infected mice in vitro, and to promote naïve CD4+ and CD8+ T cell responses in vivo ${ }^{7}$. Particularly, exosomes released from antigen presenting cells (APCs) such as dendritic cells (DCs) have emerged as potential immunotherapy due to their stability during storage, composition of immune regulatory molecules, high biocompatibility, and safety ${ }^{8,9}$. Immature DCs maintain $\mathrm{T}$ cell tolerance, whereas mature DCs have a higher number of peptide/MHC (pMHC) complexes and costimulatory molecules such as CD40 and CD80/86 interacting with CD40 ligand and CD28, respectively, on the T cell, rendering them highly effective at inducing $\mathrm{T}$ cell responses ${ }^{10}$. Herein, DC-derived exosomes can be taken up and subsequently processed by APCs or present pMHC complexes to elicit antigen-specific, MHC-restricted, T cell responses ${ }^{11}$. For instance, exosomes derived from DCs of male mice with functional pMHC complexes are able to elicit responses from naïve, transgenic, male-antigen-specific CD4+ T cells both in vivo and in vitro ${ }^{12}$. It also has been reported that exosomes secreted by virus-infected cells can elicit immune responses due to carrying viral-specific proteins and RNAs ${ }^{13}$. Montaner-Tarbes et al. demonstrated that pigs vaccinated with serumderived exosomes isolated from pigs previously infected with porcine respiratory and reproductive virus had

${ }^{1}$ Department of Veterinary Microbiology and Preventive Medicine, lowa State University, Ames, IA, USA. ${ }^{2}$ Department of Pharmaceutics, College of Pharmacy, University of Florida, Gainesville, FL, USA. ${ }^{\square}$ email: jlmcgill@iastate.edu 

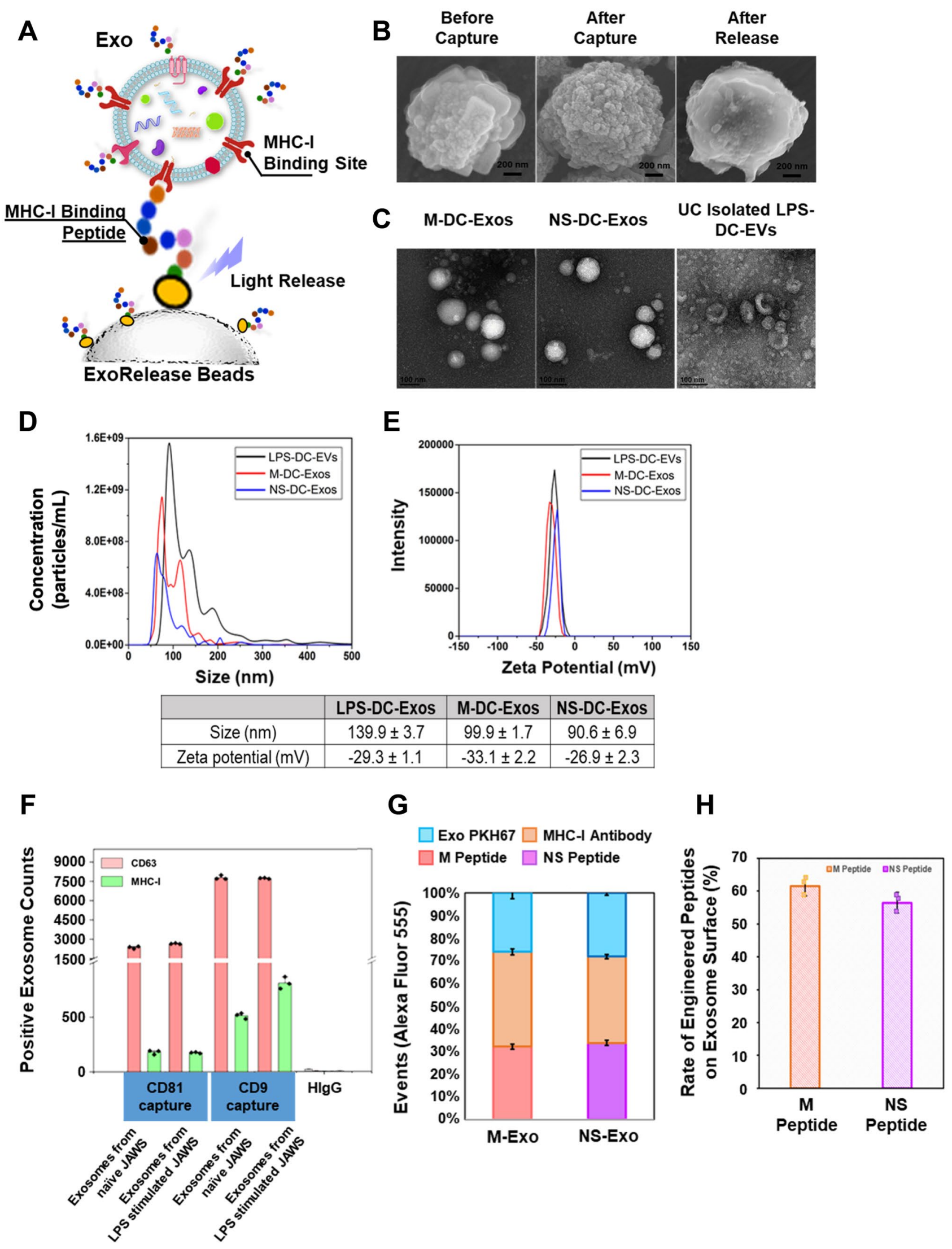
४Figure 1. Characterization of RSV-Specific Peptide Engineered DC Exosomes. (A) Schematic illustration of ExoRelease beads prepared, peptide-engineered exosomes via MHC binding peptide capture, surface MHC peptide coating, and photo-release for harvesting. (B) SEM images showing the ExoRelease bead surface morphology during the exosome capture, surface engineering, and release of intact captured exosomes. The entire surface was covered by round-shape exosomes after capture which is significantly different than the bare surface after release of intact captured exosomes. (C) TEM images showing the morphology of harvested exosomes using ExoRelease beads approach, which is in uniform size and round shape. However, ultracentrifugation isolated vesicles are more heterogeneous with substantial particle aggregates. (D) The nanoparticle tracking analysis of harvested, peptide-engineered exosomes using ExoRelease bead approach, compared with ultracentrifugation isolated vesicles without peptide engineering. Here two peptides M and NS were prepared for exosome surface engineering. The peptide engineered exosomes are in much narrower size distribution. (E) Zeta potential analysis of surface engineered exosomes compared with ultracentrifugation isolated vesicles as the control group. The zeta potential from peptide engineered exosomes were slightly changed due to the surface engineering, but still maintain the good integrity. (F) NanoView microarray was used to determine the level of MHC-I the exosomes. Exosomes from LPS stimulated JAWS expressed more MHC-I on their surface. (G) The stacked bar chart showing the percentage distribution of total exosomes, total MHC-I on exosome surface, and total RSV peptides (M and NS) bound to exosome MHC-I, which is analyzed by bead-based flow cytometry. $(\mathbf{H})$ The rate of engineered RSV peptides (M and NS) on exosome surfaces $(n=3$, RSD $<5 \%)$.

the capacity to elicit virus-specific humoral immune responses ${ }^{14}$. Exosomes carrying the spike $S$ protein from SARS-CoV have also been recently shown to elicit SARS-CoV-specific neutralizing antibody titers ${ }^{15}$. Due to the innate immunogenicity and safety and evident importance in cell signaling in vivo, an exosome-based vaccine has gained significant interest in aspects of research and clinical application.

Due to the capacity for DC-derived exosomes to elicit robust adaptive immune responses, we previously developed a microfluidic-based cell culture chip to enable real-time isolation and engineering of exosomes secreted from DCs with streamlined systems ${ }^{16}$. This showed high efficiency in generating exosomes harboring pMHC complexes, which induced in vitro proliferation of transgenic, gp100-specific CD8+ T cells in the presence of APCs ${ }^{16}$. Here, we further extended the work by investigating the potential for developing an engineered, antigenic exosome-based vaccine against a common viral infection. In this work, we further developed novel immunomagnetic nanographene particles (ExoRelease) with the function of immunocapture and on demand light-triggered release, which is suited for large scale exosome isolation and surface engineering. The streamlined workflow and simple operation for surface engineering of therapeutic peptides on DC exosomes have not been reported elsewhere. Due to the formation of pMHC-I complexes on exosome surface, this platform is amendable for highly efficient production of therapeutic peptide-tailored exosome vaccines. Exosomes were surface-engineered to harbor two well-described, immunodominant, $\mathrm{H}-2 \mathrm{D}^{\mathrm{b}}$-restricted peptides from RSV: the $\mathrm{M}_{187-195}$ and $\mathrm{NS1}_{61-75}$ peptides ${ }^{17,18}$. Their potency and immunogenicity were investigated, and we observed robust interferon-gamma (IFN- $\gamma$ ) production by activated RSV-specific CD8+ T cells ex vivo. Using the mouse model with RSV infection, we observed safe administration without any adverse effects, however, vaccination with surface-engineered exosomes did not elicit antigen-specific CD8+ T cell responses in vivo. Thus, our results show that surface engineered exosomes have the capacity to activate RSV-specific T cell responses, but further research is needed to validate their immunogenicity in vivo.

\section{Results}

Harvest and characterize RSV specific peptide engineered DC exosomes. In our previous work, we have shown that polydimethylsiloxane (PDMS) polymer made microfluidic culture chip engineered tumor antigenic DC exosomes possessed the ability to activate transgenic, antigen-specific CD8+ T cells ${ }^{16}$. Herein, we further developed a novel immunomagnetic nanographene particles (ExoRelease) with the function of immunocapture and on demand light-triggered release (Fig. 1A). The RSV-specific peptides $\left(\mathrm{M}_{187-195}\right.$ or NS1 $\left.1_{61-75}\right)$ can form the binding complex with MHC-I binding sites on the exosome surface. Particularly, DC-derived exosomes contain highly abundant MHC-I binding sites for antigenic presentation and immune activation. Here we used mature JAWS cell-derived exosomes stimulated by LPS (Lipopolysaccharide from E. coli 0111:B4). Therefore, MHC-I positive exosomes can be captured by such peptides conjugated with a photo-cleavage linker on the surface of ExoRelease particles. The unique nano-morphology of these ExoRelease particles was shown in Fig. 1B, which enables more efficient capture of exosomes than conventional beads due to much larger surface area and comparable nano-scale cavities ${ }^{19}$. After capture, the uniform, dense, and nano-sized exosomes were shown covering the entire surface of ExoRelease particles (Fig. 1B). Subsequently, the peptides either $\mathrm{M}_{187-195}$ or NS1 $1_{61-75}$ can be introduced to incubate with captured exosomes for surface engineering. The intact, engineered exosomes can be harvested via the light-triggered release. Figure 1B SEM images showed the morphology of ExoRelease particles after photo-release of intact engineered exosomes, which is clean and comparable with the morphology before capture, indicating the effective exosome isolation, surface engineering, and photo-release. TEM images of harvested engineered exosomes harboring either M or NS1 peptides were shown in Fig. 1C. The uniform, round-shape vesicles in a size range around $100 \mathrm{~nm}$ indicate the good quality of harvested exosomes. We did not observe any morphology differences between $\mathrm{M}_{187-195}$ peptide and NS1 ${ }_{61-75}$ peptide engineered exosomes. The harvested engineered exosomes showed much purer quality than the exosomes isolated from ultracentrifugation approach which displayed cell debris and aggregates (Fig. 1C). According to the nanoparticle tracking analysis, the non-engineered, LPS stimulated DC exosomes isolated by ultracentrifugation displayed a size distribution 
around 139.9 $\pm 3.7 \mathrm{~nm}$ shown in Fig. 1D. In contrast, both $\mathrm{M}_{187-195}$ and $\mathrm{NS1}_{61-75}$ peptide engineered exosomes exhibited narrower size distribution around $90-100 \mathrm{~nm}$ (Fig. 1D). The zeta potential is an important and readily measurable indicator of surface properties of EVs and exosomes, which can assess the influences from surface chemistry and bioconjugation applied ${ }^{20}$. In Fig. $1 \mathrm{E}$, we observed a slight reduction from $\mathrm{M}_{187-195}$ peptide engineered exosomes and a slight increase from $\mathrm{NS}_{61-75}$ peptide engineered exosomes. Due to the positive charge property of $\mathrm{M}_{187-195}$ peptide and the negative charge property of $\mathrm{NS1}_{61-75}$ peptide, such zeta potential changes indicate the successful surface engineering of DC exosomes with RSV-specific peptides (Fig. 1E). We also used the single-EV microarray imaging technology from NanoView to directly determine the MHC-I expression level from prepared exosomes as well as their functional markers shown in Fig. 1F. The specific antibody capture on each NanoView chip spot allows the affinity capture of exosomes based on their surface markers for further multiplexed affinity probing. Here we used CD63 and MHC-I antibodies for probing. Multiplexed fluorescence images of each antibody capture spot can derive the total counts of positive exosomes expressing MHC-I. The isotype spots (HIgG) were used as the background and non-specific control. We observed that CD9 positive exosome capture spots displayed good expression level of MHC-I, other than CD81 capture spots, which indicates that JAWS exosomes are mainly CD9 positive and LPS stimulated JAWS exosomes express more MHC-I on the surface. The CD63 is the common structural and functional proteins during exosome biogenesis, which serves as the exosome reference marker to assess the total exosomes. Our observation in Fig. 1F displayed comparable abundance between all samples and chip spots, indicating the consistent and reproducible preparation of JAWS exosomes for peptide engineering. As seen in Fig. $1 \mathrm{G}$ and $\mathrm{H}$, we used fluorescence labeling and CytoFlex flow cytometry to identify the percentage of total MHC-I on the exosome surface, as well as the percentage of total peptide bound to exosome MHC-I. By normalization, we could get the rate of engineered peptides on the exosome surface, which is about $\sim 60 \%$. Thus, the RSV peptides were sufficiently engineered on the exosome surface.

RSV specific peptide engineered DC exosomes activate RSV specific CD8 T cells ex vivo. To determine if engineered exosomes have the capacity to elicit virus-specific immune responses ex vivo, we first established a CD8 T cell-APC co-culture model (Fig. 2A). To generate RSV-specific CD8+ T cells, C57BL/6J mice were infected intranasally with RSV strain A2. On day 8 after infection, the mice were sacrificed and CD8+ T cells were isolated from the lungs and spleen by negative selection using magnetic cell separation. In our prior study, we determined that non-engineered or peptide-engineered exosomes alone are not sufficient to induce in vitro CD8 $\mathrm{T}$ cell activation, and the presence of an APC is required ${ }^{16}$. Therefore, CD11c+DCs were isolated from naïve C57BL/6J mice to serve as APC. Purified CD8+ T cells were mixed in a 5:3 ratio with naïve CD11c + dendritic cells. Engineered exosomes loaded with either RSV $\mathrm{M}_{187-195}$ (NAITNAKII) or NS1 (1-75 $_{\text {(ICPNNNIVV) }}$ peptides were added in escalating concentrations to the cell cultures. It has been previously demonstrated that exosomes secreted from activated DCs carry costimulatory molecules such as CD80, CD86 and CD40, which are necessary for activating antigen-specific CD8+ $\mathrm{T}$ cells ${ }^{10,21}$. Therefore, we hypothesized that exosomes from matured DCs could be more efficient for activating CD8+ T cells, compared to exosomes from naive DCs. To this end, T cell:DC cultures were stimulated with engineered exosomes derived from either LPS-stimulated or naïve DCs (JAWS II cell line). On day 3 of stimulation, cell culture supernatants were collected and analyzed for IFN- $\gamma$ by ELISA. As seen in Fig. 2B (the 2nd panel), $M_{187-195}$ peptide engineered exosomes derived from both immature and mature DCs induced antigen-specific IFN- $\gamma$ production by the CD8+ T cells, with no significant differences in the response to the different types of exosomes. However, with the presence of mature DCs in the co-culture, the IFN- $\gamma$ production by the CD8 + T cells was about twofold higher than that with the presence of naive DCs (Fig. 2B, the 3rd panel). A similar trend was observed with the $\mathrm{NS}_{61-75}$ peptide engineered exosomes (Fig. 2C), in which exosomes from either naïve or LPS-activated JAWSII DC cells elicited antigen-specific IFN- $\gamma$ production by the CD8+ T cells. However, the co-cultured mature DCs played an essential role for facilitating CD8+ T cell activation as indicated by the significantly increased production of IFN- $\gamma$ in the stimulated cell culture supernatants. Control wells for the T cell:DC co-cultures are shown in Fig. 2B and C (the 1st panels). Compared to mock-stimulated controls, stimulation with the $\mathrm{M}_{187-195}$ or $\mathrm{NS1}_{61-75}$ peptide only, as well as the nonspecific mitogen ConA, induced robust, antigen-specific IFN- $\gamma$. Interestingly, the concentrations of IFN- $\gamma$ induced by the engineered-exosomes was higher than that from the peptide stimulated control cultures, suggesting that the surface-engineered exosomes are very efficient activators of CD8+ T cell responses ex vivo, and could be highly immunogenic for activating RSV-specific CD8+ T cell responses in vivo.

Safety and immunogenicity of RSV specific peptide engineered DC exosomes in vivo. We next determined the safety and immunogenicity of RSV-specific peptide engineered DC exosomes in the C57BL/6 mouse model. In our initial studies, groups of mice were vaccinated subcutaneously with a mixture of both types of peptide-bearing exosomes $\left(\mathrm{M}_{187-195}\right.$ and $\left.\mathrm{NS}_{61-75}\right)$ from LPS-matured JAWSII DC cells. Mice were vaccinated with engineered exosomes only, or with engineered exosomes plus a Quil A adjuvant. Second and third booster immunizations were performed at 3-week intervals. No adverse reactions or vaccine-site reactions were observed at any time in the mice, suggesting that the engineered-exosomes are safe and well tolerated. The mice were sacrificed 3 weeks following the final immunization and assessed for antigen-specific CD8 + T cell responses in the spleen and lung. No $\mathrm{M}_{187-195}$ or $\mathrm{NS}_{61-75}$-specific CD8 + T cell responses were detected in these animals (data not shown). It is possible that exosome-immunization primed antigen-specific CD8 $\mathrm{T}$ cells, but that the responses were too low to be detected. However, an anamnestic increase in the virus-specific $\mathrm{T}$ cell response may be detectable following a viral challenge. To address this hypothesis, we studied five groups of animals by vaccinating subcutaneously with Quil A (10 $\mu \mathrm{g} / \mathrm{mouse})$, a mixture of exosomes $\left(\mathrm{M}_{187-195}\right.$ and NS1 $\left.1_{61-75}\right)$ from naïve JAWS cells $(100 \mu \mathrm{L} /$ mouse $)$, a mixture of exosomes $\left(\mathrm{M}_{187-195}\right.$ and $\left.\mathrm{NS1}_{61-75}\right)$ from naïve JAWS cells $(100 \mu \mathrm{L} /$ mouse) with Quil A, a mixture of exosomes $\left(\mathrm{M}_{187-195}\right.$ and $\left.\mathrm{NS1}_{61-75}\right)$ from LPS stimulated JAWS cells $(100 \mu \mathrm{L} /$ 


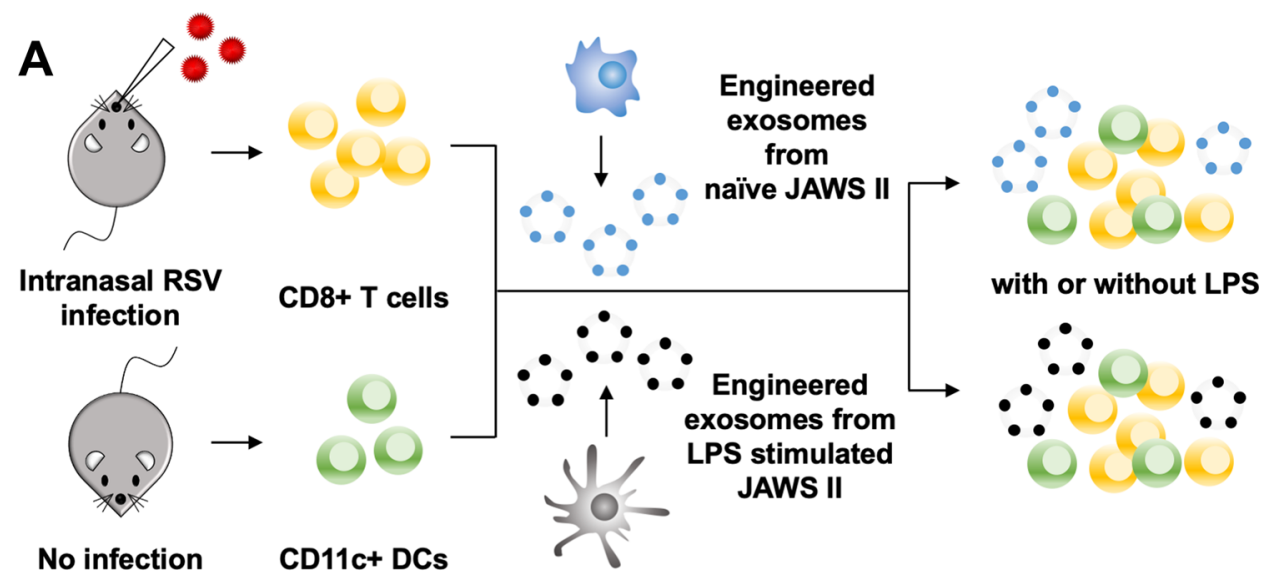

B

$M_{187-195}$ peptide on exosomes

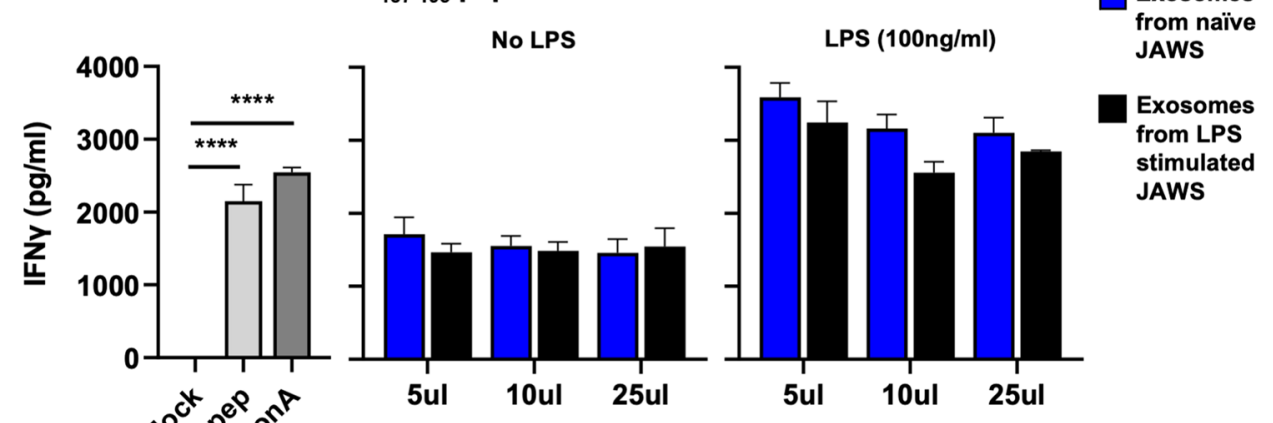

C NS1 $1_{61-75}$ peptide on exosomes

No LPS

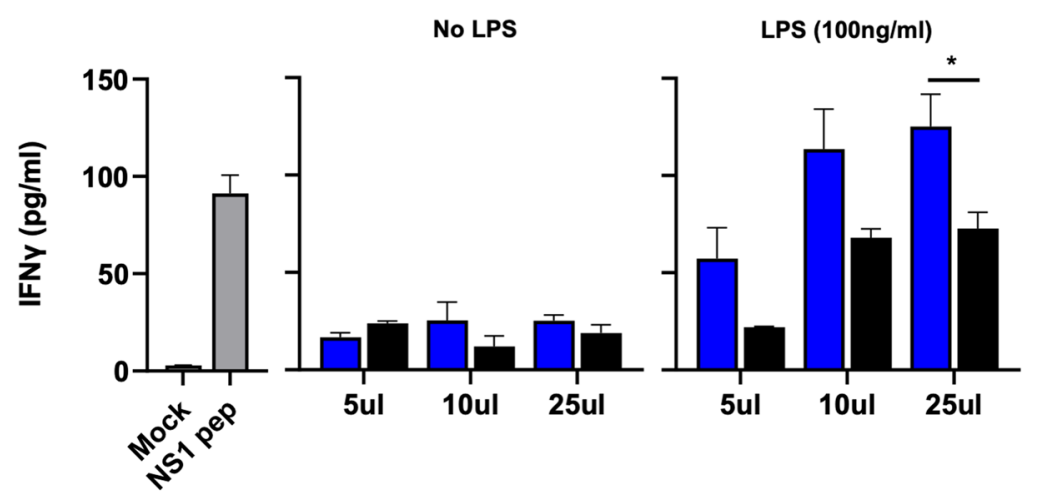

Figure 2. Engineered exosomes were immunogenic for activating RSV-specific CD8 T cells ex vivo. (A) Schematic illustration of the establishment of a DC-CD8 T cell co-culture model. C57BL/6J mice were infected intranasally with RSV strain A2 to generate RSV-specific CD8+ T cells. Magnetic-activated cell sorting (MACS) was used to sort CD8+ T cells from RSV-infected mice and CD11c + DCs from non-infected mice. CD8+ T cells and CD11c + DCs $(5: 3$ ratio) were incubated for $72 \mathrm{~h}$ with escalating concentrations $(5 \mu \mathrm{L}, 10 \mu \mathrm{L}, 25 \mu \mathrm{L})$ of exosomes engineered with $\mathrm{M}_{187-195}$ or $\mathrm{NS}_{61-75}$ peptides with or without LPS (100 ng/mL). (B, C) Cell culture supernatants were collected on day 3 of stimulation with $\mathrm{M}_{187-195}$ or $\mathrm{NS1}_{61-75}$ engineered exosomes. The samples were diluted 1:4 and analyzed by commercial ELISA for IFN- $\gamma$. Data represent means \pm SEM. ${ }^{* * *} \mathrm{p}<0.0001$ as determined by one-way ANOVA and Dunnett's multiple comparisons test. ${ }^{*} \mathrm{p}<0.05$ as determined by two-way ANOVA and Š́́dák's multiple comparisons test.

mouse), or a mixture of exosomes $\left(\mathrm{M}_{187-195}\right.$ and $\left.\mathrm{NS}_{61-75}\right)$ from LPS stimulated JAWS cells $(100 \mu \mathrm{L} / \mathrm{mouse})$ with Quil A, respectively. A booster immunization was performed two weeks later and then mice were challenged via intranasal inoculation with $10^{7}$ plaque-forming unit (PFU) of RSV strain A2. Groups of mice were sacrificed either 3 or 6 days post-infection (dpi) and their lungs and spleens were analyzed for $\mathrm{M}_{187-195}$-specific CD8 T cells by tetramer staining (Fig. 3) or for both $\mathrm{M}_{187-195}$ and $\mathrm{NS1}_{61-75}$-specific $\mathrm{T}$ cells by intracellular cytokine staining (Fig. 4). Representative $\mathrm{M}_{187-195}$ tetramer staining from 6 dpi is shown in Fig. 3A. There were no significant differences in the frequency of $\mathrm{M}_{187-195}$-specific CD8 $\mathrm{T}$ cells among groups in the lung or spleen at $3 \mathrm{dpi}$, and the 
A

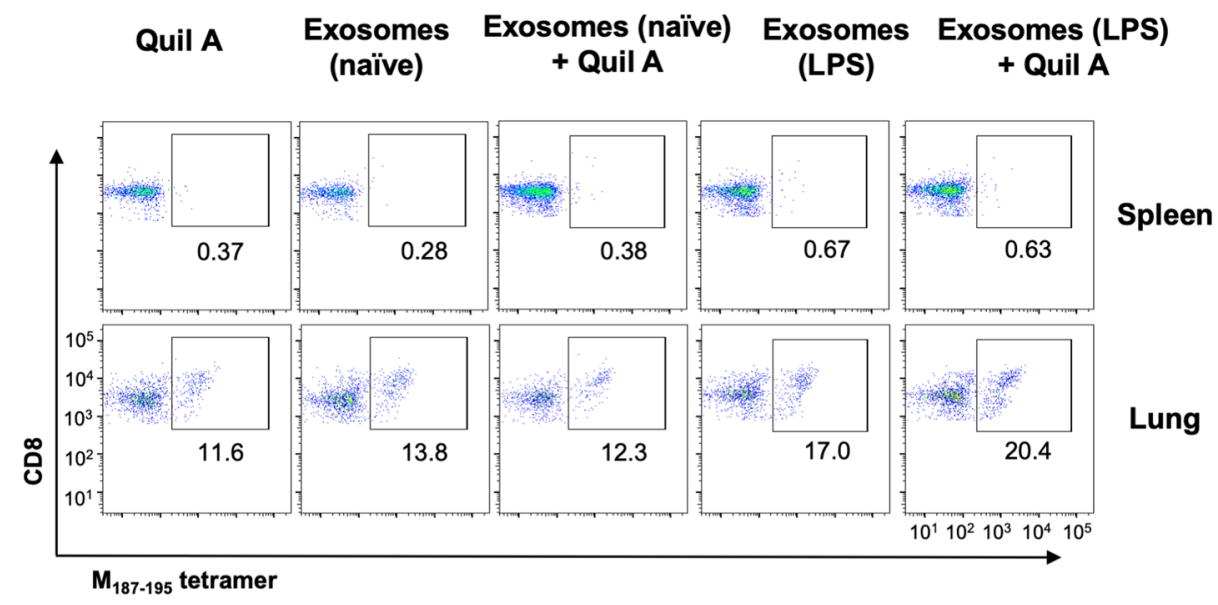

B

3 dpi

C

$6 \mathrm{dpi}$

Spleen
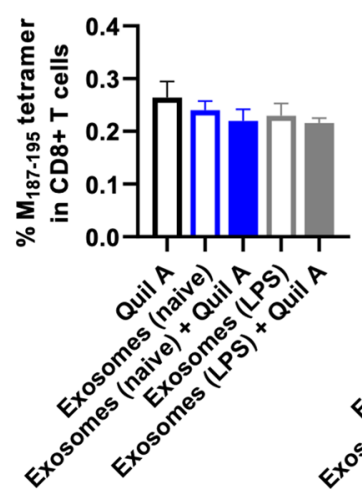

Lung

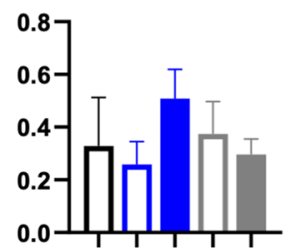

Spleen

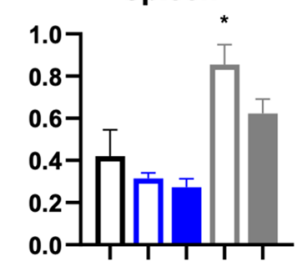

Lung
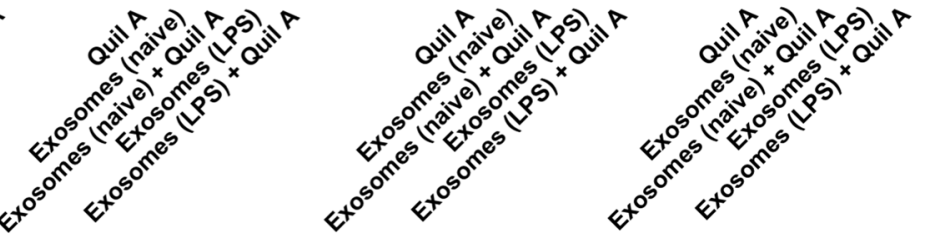

Figure 3. Confirmation of $\mathrm{M}_{187-195}$-specific CD8 T cells by tetramer staining. Groups of vaccinated mice were sacrificed either 3 or 6 days post-infection (dpi). Their lungs and spleens were stained with antibodies and analyzed by flow cytometry. (A) Representative flow cytometry plots of lungs and spleens at 6 dpi of RSV. (B, C) The antigen-specific CD8 T cells were determined based on $\mathrm{M}_{187-195}$-tetramer staining. Data are presented as means \pm SEM. ${ }^{*} \mathrm{p}<0.05$ as determined by one-way ANOVA and Holm-Šídák’s multiple comparisons test.

frequency of $\mathrm{M}_{187-195}$-specific CD8 T cells was not above background for any group, suggesting that there were no anamnestic CD8 T cell responses induced by exosome vaccination and challenge (Fig. $3 \mathrm{~B}$ ). In contrast, by day 6 post-infection, significant numbers of tetramer-positive CD8 T cells were detected in both the lungs and spleen of all groups (Fig. 3C). However, there were no differences in the frequency of cells detected in vaccinated vs. non-vaccinated animals.

Functional $\mathrm{T}$ cell responses were also measured by intracellular cytokine staining (ICS) and ELISA for IFN- $\gamma$ using antigen-recall assays. Figure 4A shows representative flow cytometry data from the lungs of a mouse that was vaccinated with exosomes from LPS-matured JAWS cells plus Quil A on 6 dpi. The frequency of CD8 T cells producing IFN- $\gamma$ in response to stimulation with the $\mathrm{M}_{187-195}$ or $\mathrm{NS1}_{61-75}$ peptides was not above background on day 3 post-infection for any group, but was detectable in all groups by day 6 (Fig. 4B). Cells from the lungs of vaccinated and infected mice were also stimulated for $72 \mathrm{~h}$ and an ELISA analysis for IFN- $\gamma$ was performed on the cell culture supernatants. Consistent with our flow cytometry results, we observed no $\mathrm{M}_{187-195}$ or NS1 $1_{61-75}$-specific IFN- $\gamma$ production from any group on $3 \mathrm{dpi}$ (Fig. 5). On $6 \mathrm{dpi}, \mathrm{M}_{187-195}$ and NS1 $1_{61-75}$-specific IFN- $\gamma$ production was detected from all groups of animals, consistent with the response to RSV challenge (Fig. 5). Lung cells collected from mice vaccinated with exosomes from naïve JAWS + Quil A (blue filled box, 3rd group) or from LPS stimulated JAWS (white filled \& gray line box, 4th group) showed significant $\mathrm{M}_{187-195}$-specific IFN- $\gamma$ production (Fig. 5A). In Fig. 5B, we noted a significant difference in $\mathrm{NS}_{61-75}$-specific IFN- $\gamma$ production between the lung cells from mice vaccinated with exosomes from naïve JAWS (white filled \& blue line box, 2nd group) and those with exosomes from LPS stimulated JAWS + Quil A (gray filled box, 5th group) (Fig. 5B). However, we observed no other differences between treatment groups. Taken together, these results suggest that RSV peptide 
A

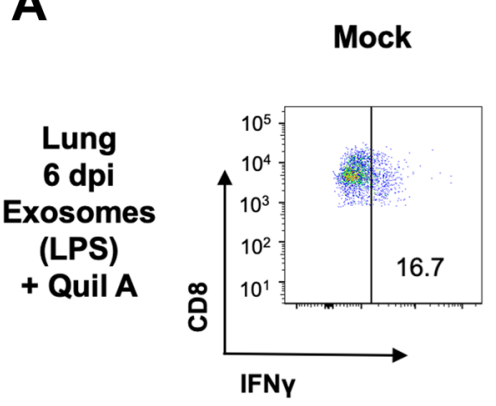

B

IFNy
$M_{187-195} \quad$ NS1 $_{61-75}$ peptide

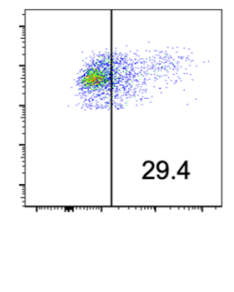

peptide

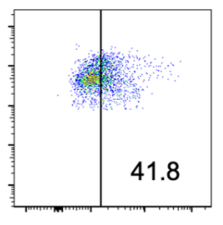

PMA/ Ionomycin

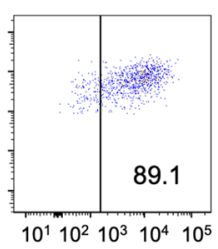

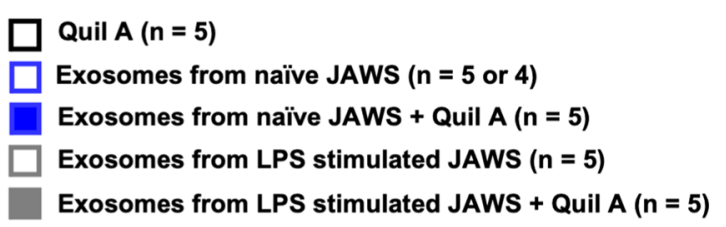

Lung

3 dpi

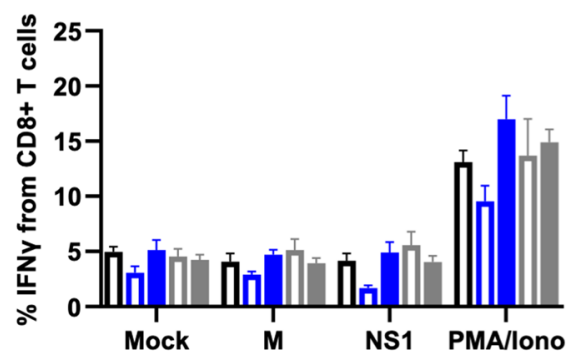

6 dpi

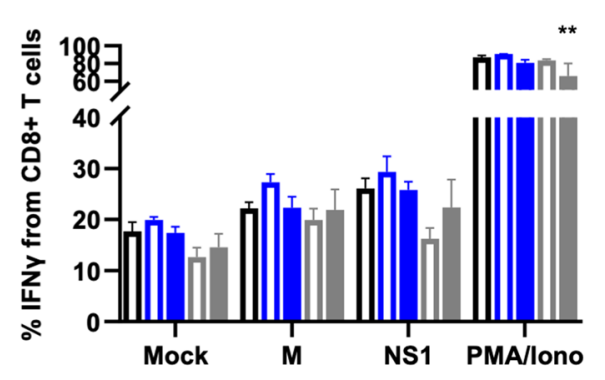

Figure 4. The production of IFN- $\gamma$ by $\mathrm{M}_{187-195}$ or $\mathrm{NS}_{61-75}$-specific T cells in the lungs. Groups of vaccinated mice were sacrificed either 3 or 6 days post-infection (dpi). Their lungs were stained with antibodies and analyzed by flow cytometry. (A) Representative flow cytometry plots showing IFN- $\gamma$ producing lungs from a group of exosomes from LPS stimulated JAWS with Quil A at 6 dpi of RSV after stimulation with M $_{187-195}$ or NS1 $1_{61-75}$ peptides. (B) Flow cytometric analysis of IFN- $\gamma$ production by lungs in the CD45+CD8+ T cell population after stimulation with the indicated conditions. Data are presented as means \pm SEM. ${ }^{* *} \mathrm{p}<0.01$ as determined by two-way ANOVA and Tukey's multiple comparisons test.

surface-engineered exosomes are highly immunogenic ex vivo; however, vaccination with the MHC-I surfaceengineered $\mathrm{M}_{187-195}$ and $\mathrm{NS}_{61-75}$ exosomes was not able to elicit exosome-induced memory $\mathrm{T}$ cell responses in vivo.

\section{Discussion}

RSV is the major cause of severe lower respiratory tract infections in infants and children worldwide, resulting in high number of hospitalization, morbidity, and mortality ${ }^{22,23}$. There is currently no vaccine available to prevent RSV infection. In this project, we aimed at developing peptide-engineered exosomes as a potential vaccine platform to elicit CD8 T cell responses against RSV. Here, we demonstrated that the novel, immunomagnetic ExoRelease particle method is effective to surface engineer DC-derived exosomes with the immunodominant $\mathrm{H}-2 \mathrm{D}^{\mathrm{b}}$-restricted $\mathrm{M}_{187-195}$ or $\mathrm{NS}_{61-75}$ peptides from RSV. The NTA analysis and Zeta potential characterization, as well as the TEM and SEM morphological study well support that we can successfully load RSV-specific peptides on the surface of DC-derived exosomes. The isolated exosomes are in a narrow and uniform size range around $100 \mathrm{~nm}$ with high integrity. Compared to currently existing methods, our ExoRelease particle method is streamlined with simple preparation protocols, which is amendable for highly efficient, large-scale production of therapeutic peptide-tailored exosome vaccines.

Our results demonstrate that DC-derived RSV-peptide engineered exosomes activate CD8 T cells ex vivo when cultured in the presence of DCs. We have previously observed that culturing non-engineered or peptide engineered-exosomes alone in the presence of CD8 T cells is not sufficient to induce T cell activation ${ }^{16}$, and the presence of an APC is required. A similar observation was made in the present study, as stimulation of RSVspecific T cells with RSV-peptide engineered exosomes did not induce ex vivo T cell activation without the 


\section{Lung}

\section{RSV $M_{187-195}$ Peptide}

3 dpi

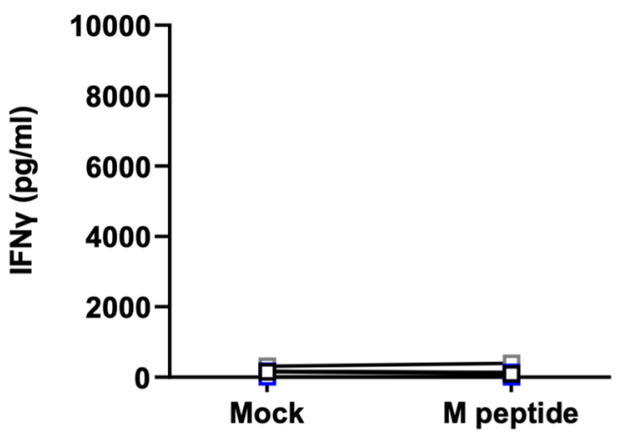

6 dpi

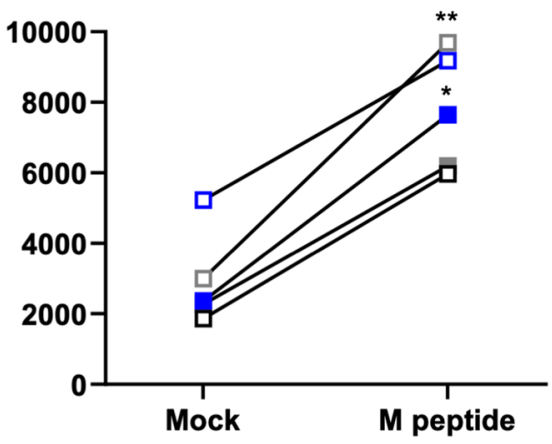

B

\section{RSV NS1 $1_{61-75}$ Peptide}

3 dpi

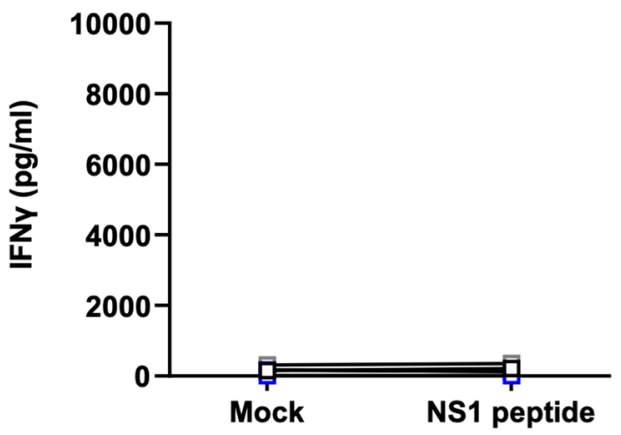

$6 \mathrm{dpi}$

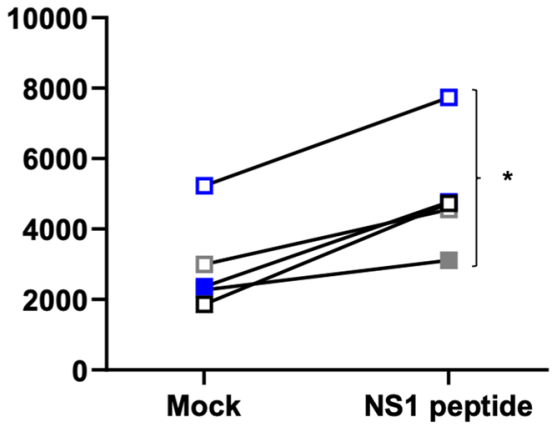

Figure 5. Vaccination with the surface engineered exosomes was not able to elicit exosome-induced memory $\mathrm{T}$ cell responses in vivo. Groups of vaccinated mice were sacrificed either 3 or 6 days post-infection (dpi). Cells from the lungs were stimulated with (A) $\mathrm{M}_{187-195}$ or (B) $\mathrm{NS}_{61-75}$ peptides for $72 \mathrm{~h}$. An ELISA analysis for IFN- $\gamma$ was performed on the cell culture supernatants. Data are presented as means \pm SEM. $(\mathbf{A}){ }^{\star} p<0.05,{ }^{* *} p<0.01$ as determined by two-way ANOVA and Šídák's multiple comparisons test. (B) ${ }^{\star} \mathrm{p}<0.05$ as determined by two-way ANOVA and Tukey's multiple comparisons test.

addition of an APC (data not shown). Thery et al. have shown that exosomes can transfer functional pMHC-II complexes to DCs to activate antigen-specific CD4+ T cells ${ }^{12}$. Thus, it seems likely that a similar transfer of pMHC-I complexes occurs in our co-culture system with CD8+ T cells. Thery et al. also demonstrated that high costimulatory molecule expression, specifically CD80 and CD86, on the APC was required to mediate efficient exosome-mediated antigen presentation and T cell activation ${ }^{12}$. In our co-culture system, the addition of LPS to the exosome:T cell:DC co-cultures resulted in significantly increased antigen-specific IFN- $\gamma$ production, supporting the requirement for matured CD11c+DCs to induce efficient antigen-presentation and T cell activation. Exosomes have similar properties or characteristics of their parental cells ${ }^{24,25}$. Given that LPS-stimulation induces costimulatory molecule expression in parental cells, peptide-engineered exosomes isolated from mature DCs could be expected carry both pMHC-I complexes and costimulatory molecules. Therefore, we initially hypothesized that exosomes prepared from LPS-matured APCs would be more immunogenic than those from immature APC, potentially negating the need for an additional external stimuli to activate the APC in the cocultures. However, we observed no differences in the quantity of antigen-specific IFN- $\gamma$ elicited from the T cells that were stimulated with exosomes from naïve DCs versus LPS-matured DCs. Thus, from our data, the presence 
of mature host APC to present exosome-derived pMHC complexes is the most important factor regulating the ex vivo response, rather than the activation state of the exosome-producing host cell.

Given their immunogenicity ex vivo, we expected that surface-engineered exosomes would have the capacity prime in vivo CTL responses. However, vaccination with peptide-engineered exosomes failed to elicit antigenspecific CD8 $\mathrm{T}$ cell responses in the animal, even when a prime-boost regimen was used. Andre et al. made a similar observation using DC-derived exosomes pulsed with a melanoma-derived CTL epitope ${ }^{26}$. In their work, inoculation with as high as $10^{11}$ peptide-pulsed exosomes failed to elicit an antigen-specific CD8 T cell response. As we observed in Fig. $1 \mathrm{G}$ and $\mathrm{H}$, our engineered exosomes carry robust quantities of pMHC complexes, thus the dose that we administered offers sufficient antigen quantity for $\mathrm{T}$ cell priming. However, free exosomes have been estimated to be as much as 20 -fold less efficient at stimulating T cell responses than their parent $\mathrm{APC}^{27}$. This weak stimulatory ability may be due to small size and wide vesicle dispersion ${ }^{28}$. In vivo, it seems likely that injected exosomes may be internalized by surrounding cells, thus diluting the availability of pMHC complexes for antigen-presentation. In vitro, we demonstrate that engineered-exosomes require the presence of an APC for efficient T cell activation. Others have shown that the inclusion of an APC in vivo similarly enhances the T cell priming capacity of exosomes. As an example, Andre et al. determined that loading of exosomes onto LPSmatured DC prior to injection significantly increased the capacity for CD8 T cell activation and differentiation in vivo ${ }^{26}$. Interestingly, however, Chaput et al. showed that the necessity for DC-loading of the exosomes could be overcome by the appropriate adjuvant. Mixing peptide-loaded exosomes with the TLR9 agonist, CpG ODN immediately prior to injection in the footpad enabled the exosomes to activate naïve, CD8 T cells in vivo ${ }^{29}$. Quil A was selected as an adjuvant in our studies. Quil A is a widely used saponin adjuvant that is known to stimulate balanced cellular and humoral immune responses, and to induce the generation of cytotoxic CD8 T cell responses ${ }^{30}$. It was our expectation that Quil A, like CpG, could enhance the immunogenicity of the peptide engineered exosomes in our system. However, inclusion of Quil A had no effects in vivo. Consistent with our results, Chaput et al. evaluated several other conventional adjuvants, including Montanide (ISA 720), which also failed to elicit exosome-induced CD8 T cell responses in vivo ${ }^{29}$. Only agonists for TLR3 (poly(I:C)) and TLR9 appeared to substitute for exosome-loaded DC in this system ${ }^{29}$. The threshold for activation of naïve CD8 T cells is known to be much higher than that of effector or memory CD8 T cells, thus further optimization may be required to overcome the naïve $\mathrm{T}$ cell activation threshold with peptide-engineered exosomes ${ }^{31-34}$. Additional factors such as cytokine environment and TLR crosslinking have been shown to reduce the threshold for naïve CD8 T cell activation ${ }^{31-33}$. This observation is consistent with that of Chaput et al., showing the importance of pattern recognition receptor engagement ${ }^{29}$. Recently the importance of robust innate immune activation on the induction of potent and long-lived memory $\mathrm{T}$ cell responses has also come to light ${ }^{35}$. In addition to their effect on adaptive immune cells, many prime-boost regimens have been shown to activate more efficient innate immune cells or engage innate immune memory, leading to more robust innate-adaptive immune cell cross-talk. In the current approach, we did not utilize an adjuvant or stimulant that would be expected to prime or boost innate immune memory, thus possibly leading to suboptimal efficacy of our peptide-engineered exosome vaccine. Our own future studies will focus on the incorporation of $\mathrm{CpG}$ or other potential TLR agonists that could be administered with the engineered exosomes to induce optimum innate immune activation and naïve $\mathrm{T}$ cell activation.

In addition to the consideration of additional adjuvants or innate immune stimulants, our future studies will further select the optimum antigenic payload for use in an engineered-exosome vaccine. The peptides selected for this study are well described, immunodominant CD8 T cell epitopes from RSV, and hence clear targets for vaccine development. However, peptides alone are not highly immunogenic. Although the strategic use of adjuvant may be sufficient to overcome this limitation, use of a larger protein fragment from RSV may prove more effective. Naslund et al. demonstrated that pMHC-I-loaded exosomes were poorly immunogenic, but loading the exosomes with a full-length protein elicited strong CD8 T cell responses in vivo ${ }^{36}$. The authors also demonstrated that CD4 $\mathrm{T}$ cell help was required for induction of exosome-induced CD8 T cell responses. In CD4 $\mathrm{T}$ cell deficient animals, even exosomes loaded with full-length protein failed to activate CD8 T cell responses. Naslund et al. also showed that B cells were required for processing the exosome-loaded proteins for CD8 and $\mathrm{CD} 4 \mathrm{~T}$ cell activation ${ }^{36}$. Thus, induction of a robust immune response by exosomes likely involves complex interactions between multiple cell types. Loading exosomes with a larger protein fragment from RSV, coupled with an appropriate TLR agonist, may be the best approach for optimizing an engineered-exosome vaccine for prevention of a viral disease such as RSV.

In conclusion, we demonstrate that surface engineered exosomes successfully activated RSV-specific CD8 $\mathrm{T}$ cells ex vivo; however, injection of $\mathrm{M}_{187-195}$ or $\mathrm{NS}_{61-75}$ engineered exosomes failed to prime virus-specific CD8 T cell responses in vivo. Importantly, we did not observe any adverse reactions after in vivo injection of surface-engineered exosomes. Further research is required to optimize the use of surface-engineered exosomes as a vaccine platform for use against viral diseases such as RSV.

\section{Methods}

Exosome isolation, therapeutic peptide surface engineering, and characterization. The proprietary fabrication of ExoRelease immunomagnetic nanographene particles produces flower-like nano pompoms morphology with photo click chemistry, which has been licensed by Clara Biotech Inc. The MHC binding peptide $M$ with sequence NAITNAKII (RSV $\mathrm{M}_{187-195}$ ) and NS1 peptide with sequence ICPNNNIVV (RSV $\mathrm{NS}_{61-75}$ ) were synthesized by GenScript, confirming $>95 \%$ purity by high performance liquid chromatography (HPLC). These peptides conjugated onto ExoRelease particles respectively via NHS chemistry for affinity capture of MHC-I positive exosomes. The exosome isolation and surface engineering procedures were performed by incubating $100 \mathrm{~mL}$ of MHC binding peptide ExoRelease particles with $6 \mathrm{~mL}$ of cell culture medium at $4{ }^{\circ} \mathrm{C}$ overnight and shielded from light for MHC-I positive exosome capture and isolation. The solution tubes were 
placed onto the magnet for $1 \mathrm{~min}$, then supernatant was discarded. Afterward, the particles were resuspended into $200 \mathrm{~mL}$ of $1 \times$ phosphate buffered saline (PBS) buffer. Next, $15 \mathrm{~mL}$ of a solution containing $\beta 2$-microglobulin $(20 \mu \mathrm{g} / \mathrm{mL})$ and peptide $(100 \mu \mathrm{g} / \mathrm{mL}$, either M peptide or NS1 peptide) for surface binding to form pMHC-I complex, was placed in a glass vial to incubate with rotation for $1 \mathrm{~h}$ at room temperature. The ExoRelease particles were separated from the reaction mixture by placing close to a magnetic bar for 1 min and discarding the supernatant; then the ExoRelease particles with surface engineered exosomes were washed three times with $1 \times$ cool PBS buffer. Subsequently, exosomes were released from particles through photo-cleavage using a LED UV head at $365 \mathrm{~nm}$ wavelength exposed for $15 \mathrm{~min}\left(\sim 6 \mathrm{~mW} / \mathrm{cm}^{2}\right)$. The conjugated PEG photo-cleavable linker with NHS moiety on bead surface allows the bond cleavage under light exposure to release intact, engineered peptide exosomes on demand, which further ensures the specificity for harvesting MHC-peptide modified exosomes. Harvested exosomes were characterized using the nanoparticle tracking analysis (NTA, Nano-Sight LM10, Malvern Panalytical) to determine size distribution and particle concentration of engineered exosomes. NTA post-acquisition parameters were adjusted to a screen gain of 10.0 and a detection threshold to 5. Standard $100 \mathrm{~nm}$ nanoparticles were used for calibration. Samples were diluted in $1 \times$ PBS before every measurement and repeated for five times. The zeta potential was measured using ZetaVeiw (Particle Metrix).

NanoView microarray for characterization of exosome MHC-I expression level was conducted per standard protocols provided by NanoView Biosciences (Brighton, MA). Processed JAWS cell culture media was diluted appropriately using the EV binding buffer (solution A, pH 7.4), then $35 \mu \mathrm{L}$ of diluted sample was dropped on microarray chips for incubation overnight. Each three chip spots were pre-coated with capture antibodies CD81 (clone Eat-2, mouse, BioLegend) and CD9 (clone MZ3, mouse, BioLegend), and negative controls HIgG (clone HTK888, mouse, BioLegend). Microarray chips were washed four times with solution A at $150 \mathrm{rpm} / \mathrm{min}, 3 \mathrm{~min}$ for each time. After washing, $300 \mu \mathrm{L}$ blocking solution was incubated with each chip for $1 \mathrm{~h}$ at room temperature and protected from light, which contains two detection antibodies including $0.6 \mu \mathrm{L}$ Alexa Fluor 647 -conjugated CD63 (clone NVG-2, mouse, BioLegend) and 2.5 $\mu \mathrm{L}$ PE-conjugated MHC class I (clone AF6-88.5.5.3, mouse, Invitrogen). Subsequently, microarray chips were washed again with solution A, solution B and distilled water, then chips were kept air dry for imaging by ExoView R100 (NanoView Biosciences) equipped with $40 \times$ objective lens (Olympus, Japan). Data was analyzed and quantified using off-line ExoViewer3 EAP_v3 software.

The bead-based flow cytometry analysis (CytoFlex, Beckman) was performed to characterize our ExoRelease particle captured exosomes and their surface engineering with RSV peptides. The exosomes were labeled by PKH67 (Sigma-Aldrich) per vendor's instruction. The RSV peptides (M and NS) were labeled by Alexa Fluor 555 (Thermo Fisher).

Characterization of engineered exosomes using SEM and TEM imaging. For scanning electron microscope observation, exosome bound particles were resuspended in $200 \mu \mathrm{L}$ PBS solution and washed 2 times with pure water. $5 \mu \mathrm{L}$ exosome bound particle solution was added to clean silicon chips and immobilized by drying under a ventilation hood. Samples on silicon chips were mounted on a SEM stage by carbon paste with $30 \mathrm{~s}$ sputtering coating. SEM imaging was performed under low beam energies (7 kV) with $10 \mathrm{nA}$ on Hitachi SU8230 field emission scanning electron microscope.

For TEM imaging, $\sim 5 \mu \mathrm{L}$ of harvested exosomes were dropped onto formvar carbon coated copper Grid 200 mesh (Electron Microscopy Sciences) for $5 \mathrm{~min}$ followed by $3 \mathrm{~min}$ of negative staining with $2 \%$ aqueous uranyl acetate. Excess liquid was blotted by a filter paper. Total grid preparation was performed at room temperature until completely air-dried under a ventilation hood for $25 \mathrm{~min}$. The imaging was performed immediately after fixation using the Tecnai G2 Spirit TWIN Transmission Electron Microscope at $120 \mathrm{kV}$.

RSV isolation. Human epithelial type 2 (HEp-2) cells (ATCC, CCL-23) were maintained in complete minimal essential media (cMEM), composed of MEM medium (Gibco) containing $10 \%$ fetal bovine serum (FBS, Gibco), $2 \mathrm{mM}$ L-glutamine (Gibco), 1\% antibiotic antimycotic (Gibco), and $1 \mathrm{mM}$ sodium pyruvate (Gibco) until they are approximately 80 to $90 \%$ confluent. RSV strain A2 (ATCC, VR-1540) was inoculated into the HEp-2 cells with serum-free MEM. After $2 \mathrm{~h}$ of virus inoculation, the supernatant was removed, and cMEM was added. For storage, RSV infected HEp- 2 cells were frozen at $-80^{\circ} \mathrm{C}$ from 48 to $72 \mathrm{~h}$ after infection. Virusinfected HEp- 2 cells were thawed, and cultures were centrifuged at $3200 \times g$ for $15 \mathrm{~min}$ at $4{ }^{\circ} \mathrm{C}$ to remove cell debris. Virus supernatants were collected and added to the polyethylene glycol 8000 in NT buffer containing $150 \mathrm{mM} \mathrm{NaCl}$ and $50 \mathrm{mM}$ Tris- $\mathrm{HCl}(10 \% \mathrm{w} / \mathrm{v})$. The supernatants were incubated at $4{ }^{\circ} \mathrm{C}$ for an hour with stirring and then centrifuged to precipitate the RSV at $3200 \times \mathrm{g}$ for $40 \mathrm{~min}$ at $4{ }^{\circ} \mathrm{C}$. The virus was suspended in $3 \%$ sucrose Dulbecco's Modified Eagle Medium (DMEM, Gibco) and stored at $-80^{\circ} \mathrm{C}$. HEp- 2 cells were inoculated with tenfold serial dilutions of RSV in a 96 well flat bottom plate. The titration was measured by cytopathic effects. The Reed and Muench calculation was used to get the virus titer.

Animal study and ex vivo experiments. All mice procedures were in accordance with the relevant guidelines and regulations, and experimental protocols were approved by the Iowa State University Institutional Animal Care and Use Committee (IACUC) 18-074. Additionally, all animal experiments were conducted in compliance with the ARRIVE (Animal Research: Reporting of In Vivo Experiments) guidelines. Six to seven week old C57BL/6J female mice were purchased from Jackson Laboratories. Mice were challenged intranasally with RSV. Animals were anesthetized with isoflurane prior to intranasal inoculation with $10^{7} \mathrm{PFU}$ RSV in $60 \mu \mathrm{L}$ $3 \%$ sucrose in DMEM and were sacrificed on day 8 post-infection by carbon dioxide $\left(\mathrm{CO}_{2}\right)$ asphyxiation, and then the lung tissues were harvested. Five RSV infected C57BL/ 6 mice were euthanized and spleens and lungs were collected for CD8+ T cells. Another five non-infected C57BL/6 (naïve) mice were euthanized and spleens were collected for CD11c+cells. Magnetic activated cell sorting (MACS) was used to sort CD8+ T cells and 
CD11c+ DCs according to the manufacturer's instructions (Miltenyi Biotec). The exosome:T cell:DC co-culture model was adapted from previously described methods ${ }^{16}$. CD8+ T cells and CD11c+DC cells (5:3 ratio) were incubated for $72 \mathrm{~h}$ with escalating concentration $(5 \mu \mathrm{L}, 10 \mu \mathrm{L}, 25 \mu \mathrm{L})$ of exosomes engineered with $\mathrm{M}_{187-195}$ or $\mathrm{NS}_{61-75}$ peptides with or without LPS $(100 \mathrm{ng} / \mathrm{mL}$, InvivoGen). Cells were cultured in complete RPMI (cRPMI), composed of RPMI 1640 medium (Gibco) containing 10\% FBS, 2 mM L-glutamine, 1\% antibiotic antimycotic, and $1 \mathrm{mM}$ sodium pyruvate. cRPMI was used as a negative control, and M peptide $(5 \mu \mathrm{g} / \mathrm{mL}$, GenScript), NS1 peptide $(5 \mu \mathrm{g} / \mathrm{mL}$, GenScript), and ConA ( $5 \mu \mathrm{g} / \mathrm{mL}$, MP Biomedicals) were used as positive controls.

Exosome immunization for challenging RSV infected mouse model. Five mice per group were immunized (prime-boost) subcutaneously with $100 \mu \mathrm{L}\left(1.7 \times 10^{9}\right.$ particles $\left./ \mathrm{mL}\right)$ of engineered exosomes harboring either M or NS1 peptides on their surface with or without Quil A adjuvants $(10 \mu \mathrm{g} / \mathrm{mouse})$. There were 2 weeks gap between prime and boost vaccination. Mice were challenged intranasally with RSV, 2 weeks after boost. The same protocols as ex vivo experiments were used to inoculate the virus and sacrifice the mice. They were anesthetized with isoflurane prior to intranasal inoculation with $10^{7} \mathrm{PFU}$ of RSV in $60 \mu \mathrm{L} 3 \%$ sucrose in DMEM. Mice were sacrificed on day 3 or 6 post-infection by carbon dioxide $\left(\mathrm{CO}_{2}\right)$ asphyxiation and lung tissues were harvested (same as ex vivo). Lung cells were stimulated and incubated with cRPMI media, M peptide $(5 \mu \mathrm{g} /$ $\mathrm{mL}$ ), and NS1 peptide $(5 \mu \mathrm{g} / \mathrm{mL})$. Positive controls used were PMA (50 ng/mL, Sigma-Aldrich) and Ionomycin $(1 \mu \mathrm{g} / \mathrm{mL}$, MP Biomedicals) for flow cytometry and ConA $(5 \mu \mathrm{g} / \mathrm{mL})$ for ELISA.

Lung cell isolation. The lungs were diced and digested by collagenase type I ( $3 \mathrm{mg} / \mathrm{mL}$, Gibco) in cRPMI for $30 \mathrm{~min}$ at $37^{\circ} \mathrm{C}$. After incubation, the tissues were processed through a $70 \mu \mathrm{m}$ strainers to prepare a single cell suspension. The cell suspension was washed twice with cRPMI at $1500 \mathrm{rpm}$ for $5 \mathrm{~min}$ at $4{ }^{\circ} \mathrm{C}$.

Tetramer and intracellular cytokine staining (ICS). After 12-h incubation with stimuli followed by 6-h incubation with Brefeldin A (Becton Dickinson Biosciences) for all wells and PMA/Ionomycin only for positive controls, virus-specific T cells were detected with tetramers of $\mathrm{H}-2 \mathrm{D}^{\mathrm{b}} \mathrm{RSV} \mathrm{M}_{187-195}$ peptide. Cells were stained with fluorochrome-conjugated antibodies against FITC anti-mouse CD45 (clone 30-F11, mouse, eBioscience,), PerCP/Cyanine5.5 anti-mouse CD8a (clone 53-6.7, mouse, BioLegend,), APC H-2D ${ }^{\mathrm{b} S V} \mathrm{M}_{187-195}$ tetramer (MBL), and aqua live/dead fixable dead cell stain (Invitrogen) for $30 \mathrm{~min}$ at $4{ }^{\circ} \mathrm{C}$. Cells were fixed and permeabilized according to the manufacturer's instructions (Becton Dickinson Biosciences) and stained with APC/Cyanine7 fluorochrome-conjugated anti-mouse IFN- $\gamma$ antibodies (clone XMG1.2, mouse, BioLegend) for $30 \mathrm{~min}$ at room temperature. After staining, cells were washed and analyzed by flow cytometry. Cells were analyzed on a Becton Dickinson FACSCanto. Data were analyzed by using FlowJo software version 10.7.1 (FlowJo, LLC). A representative gating strategy for analysis of tetramer and intracellular cytokine staining is included in Supplementary Fig. 1.

ELISAs. Mouse IFN- $\gamma$ DuoSet ELISA Development kit was purchased from R\&D systems and ELISAs were performed according to manufacturer's instructions. Sandwich ELISAs were used to quantify mouse IFN- $\gamma$ in cell culture supernatants. As previously described, lung cells were stimulated and incubated with cRPMI media, M peptide $(5 \mu \mathrm{g} / \mathrm{mL})$, NS1 peptide $(5 \mu \mathrm{g} / \mathrm{mL})$, and ConA $(5 \mu \mathrm{g} / \mathrm{mL})$. The supernatants were collected after $72-\mathrm{h}$ or 5-day stimulation with those stimuli. The supernatant samples were diluted 1:4 with reagent diluent buffer. All samples and standards were plated in duplicates. The results of ELISA are representative of three independent ex vivo experiments and that of one in vivo experiment.

Statistics. Results were shown as averages \pm standard errors of the mean (SEM). Statistical analysis was determined by one-way and two-way Analysis of Variance (ANOVA) using Prism software version 9.0.0 (GraphPad Software, LLC).

Received: 1 June 2021; Accepted: 13 October 2021

Published online: 01 November 2021

\section{References}

1. Shi, T. et al. Global, regional, and national disease burden estimates of acute lower respiratory infections due to respiratory syncytial virus in young children in 2015: A systematic review and modelling study. Lancet 390, 946-958. https://doi.org/10.1016/ S0140-6736(17)30938-8 (2017).

2. Aheget, H. et al. Exosomes: Their role in pathogenesis, diagnosis and treatment of diseases. Cancers https://doi.org/10.3390/cance rs13010084 (2020)

3. Ludwig, A. K. \& Giebel, B. Exosomes: Small vesicles participating in intercellular communication. Int. J. Biochem. Cell Biol. 44, 11-15. https://doi.org/10.1016/j.biocel.2011.10.005 (2012).

4. Ruan, S. et al. Extracellular vesicles as an advanced delivery biomaterial for precision cancer immunotherapy. Adv. Healthc. Mater. 1, e2100650. https://doi.org/10.1002/adhm.202100650 (2021).

5. Raposo, G. et al. B lymphocytes secrete antigen-presenting vesicles. J. Exp. Med. 183, 1161-1172. https://doi.org/10.1084/jem. 183.3.1161 (1996).

6. Lugini, L. et al. Immune surveillance properties of human NK cell-derived exosomes. J. Immunol. 189, 2833-2842. https://doi. org/10.4049/jimmunol.1101988 (2012).

7. Giri, P. K. \& Schorey, J. S. Exosomes derived from M. Bovis BCG infected macrophages activate antigen-specific CD4+ and CD8+ T cells in vitro and in vivo. PLoS ONE 3, e2461. https://doi.org/10.1371/journal.pone.0002461 (2008). 
8. Andre, F., Escudier, B., Angevin, E., Tursz, T. \& Zitvogel, L. Exosomes for cancer immunotherapy. Ann. Oncol. 15(Suppl 4), 141-144. https://doi.org/10.1093/annonc/mdh918 (2004).

9. Pitt, J. M. et al. Dendritic cell-derived exosomes for cancer therapy. J. Clin. Invest. 126, 1224-1232. https://doi.org/10.1172/JCI81 137 (2016).

10. Segura, E., Amigorena, S. \& Théry, C. Mature dendritic cells secrete exosomes with strong ability to induce antigen-specific effector immune responses. Blood Cells Mol. Dis. 35, 89-93. https://doi.org/10.1016/j.bcmd.2005.05.003 (2005).

11. Pitt, J. M. et al. Dendritic cell-derived exosomes as immunotherapies in the fight against cancer. J. Immunol. 193, 1006-1011. https://doi.org/10.4049/jimmunol.1400703 (2014).

12. Théry, C. et al. Indirect activation of naïve CD4+ T cells by dendritic cell-derived exosomes. Nat. Immunol. 3, 1156-1162. https:// doi.org/10.1038/ni854 (2002).

13. Crenshaw, B. J., Gu, L., Sims, B. \& Matthews, Q. L. Exosome biogenesis and biological function in response to viral infections. Open Virol. J. 12, 134-148. https://doi.org/10.2174/1874357901812010134 (2018).

14. Montaner-Tarbes, S. et al. Targeted-pig trial on safety and immunogenicity of serum-derived extracellular vesicles enriched fractions obtained from Porcine Respiratory and Reproductive virus infections. Sci. Rep. 8, 17487. https://doi.org/10.1038/s41598018-36141-5 (2018).

15. Kuate, S., Cinatl, J., Doerr, H. W. \& Uberla, K. Exosomal vaccines containing the S protein of the SARS coronavirus induce high levels of neutralizing antibodies. Virology 362, 26-37. https://doi.org/10.1016/j.virol.2006.12.011 (2007).

16. Zhao, Z., McGill, J., Gamero-Kubota, P. \& He, M. Microfluidic on-demand engineering of exosomes towards cancer immunotherapy. Lab. Chip. 19, 1877-1886. https://doi.org/10.1039/c8lc01279b (2019).

17. Rutigliano, J. A., Rock, M. T., Johnson, A. K., Crowe, J. E. \& Graham, B. S. Identification of an H-2D(b)-restricted CD8+ cytotoxic T lymphocyte epitope in the matrix protein of respiratory syncytial virus. Virology 337, 335-343. https://doi.org/10.1016/j.virol. 2005.04.032 (2005).

18. Schmidt, M. E. \& Varga, S. M. Identification of novel respiratory syncytial virus CD4. Immunohorizons 3, 1-12. https://doi.org/ 10.4049/immunohorizons.1800056 (2019).

19. He, N. et al. Nano pom-poms prepared highly specific extracellular vesicles expand the detectable cancer biomarkers. BioRxiv (2021).

20. Midekessa, G. et al. Zeta potential of extracellular vesicles: Toward understanding the attributes that determine colloidal stability. ACS Omega 5, 16701-16710. https://doi.org/10.1021/acsomega.0c01582 (2020).

21. Wahlund, C. J. E. et al. Exosomes from antigen-pulsed dendritic cells induce stronger antigen-specific immune responses than microvesicles in vivo. Sci. Rep. 7, 17095. https://doi.org/10.1038/s41598-017-16609-6 (2017).

22. Stein, R. T. et al. Respiratory syncytial virus hospitalization and mortality: Systematic review and meta-analysis. Pediatr. Pulmonol. 52, 556-569. https://doi.org/10.1002/ppul.23570 (2017).

23. Nair, H. et al. Global burden of acute lower respiratory infections due to respiratory syncytial virus in young children: A systematic review and meta-analysis. Lancet 375, 1545-1555. https://doi.org/10.1016/S0140-6736(10)60206-1 (2010).

24. Hu, Q. et al. Clinical applications of exosome membrane proteins. Precis. Clin. Med. 3, 54-66. https://doi.org/10.1093/pcmedi/ pbaa007 (2020).

25. Zhang, Y., Liu, Y., Liu, H. \& Tang, W. H. Exosomes: Biogenesis, biologic function and clinical potential. Cell. Biosci. 9, 19. https:// doi.org/10.1186/s13578-019-0282-2 (2019).

26. André, F. et al. Exosomes as potent cell-free peptide-based vaccine. I. Dendritic cell-derived exosomes transfer functional MHC class I/peptide complexes to dendritic cells. J. Immunol. 172, 2126-2136. https://doi.org/10.4049/jimmunol.172.4.2126 (2004).

27. Vincent-Schneider, H. et al. Exosomes bearing HLA-DR1 molecules need dendritic cells to efficiently stimulate specific T cells. Int. Immunol. 14, 713-722. https://doi.org/10.1093/intimm/dxf048 (2002).

28. Robbins, P. D. \& Morelli, A. E. Regulation of immune responses by extracellular vesicles. Nat. Rev. Immunol. 14, 195-208. https:// doi.org/10.1038/nri3622 (2014).

29. Chaput, N. et al. Exosomes as potent cell-free peptide-based vaccine. II. Exosomes in CpG adjuvants efficiently prime naive Tc1 lymphocytes leading to tumor rejection. J. Immunol. 172, 2137-2146. https://doi.org/10.4049/jimmunol.172.4.2137 (2004).

30. Cibulski, S. P. et al. Novel ISCOMs from Quillaja brasiliensis saponins induce mucosal and systemic antibody production, T-cell responses and improved antigen uptake. Vaccine 34,1162-1171. https://doi.org/10.1016/j.vaccine.2016.01.029 (2016).

31. Mercier, B. C., Cottalorda, A., Coupet, C. A., Marvel, J. \& Bonnefoy-Bérard, N. TLR2 engagement on CD8 T cells enables generation of functional memory cells in response to a suboptimal TCR signal. J. Immunol. 182, 1860-1867. https://doi.org/10.4049/ jimmunol.0801167 (2009).

32. Cottalorda, A. et al. TLR2 engagement on CD8 T cells lowers the threshold for optimal antigen-induced T cell activation. Eur. J. Immunol. 36, 1684-1693. https://doi.org/10.1002/eji.200636181 (2006).

33. Wijesundara, D. K., Kumar, S., Alsharifi, M., Müllbacher, A. \& Regner, M. Antigen-specific activation thresholds of CD8+ T cells are independent of IFN-I-mediated partial lymphocyte activation. Int. Immunol. 22, 757-767. https://doi.org/10.1093/intimm/ dxq064 (2010).

34. Zikherman, J. \& Au-Yeung, B. The role of T cell receptor signaling thresholds in guiding $\mathrm{T}$ cell fate decisions. Curr. Opin. Immunol. 33, 43-48. https://doi.org/10.1016/j.coi.2015.01.012 (2015).

35. Palgen, J. L. et al. Optimize prime/boost vaccine strategies: trained immunity as a new player in the game. Front. Immunol. 12, 612747. https://doi.org/10.3389/fimmu.2021.612747 (2021).

36. Näslund, T. I., Gehrmann, U., Qazi, K. R., Karlsson, M. C. \& Gabrielsson, S. Dendritic cell-derived exosomes need to activate both $\mathrm{T}$ and B cells to induce antitumor immunity. J. Immunol. 190, 2712-2719. https://doi.org/10.4049/jimmunol.1203082 (2013).

\section{Acknowledgements}

The authors wish to thank Ronald Kardelis, Taylor Springer, Michelle Thacker, and Laboratory Animal Resources for their attentive care of the mice. The authors would like to acknowledge Shawn Rigby to acquire data from flow cytometry at flow cytometry facility. The authors also wish to acknowledge Dr. David Verhoeven for his help to purify RSV strain A2. The project was supported by USDA Agriculture and Food Research Initiative Award 2017-67021-28898 to Dr. Mei He.

\section{Author contributions}

S.R. prepared peptide engineered exosomes and characterized by NTA and TEM. Z.G. prepared and isolated exosomes and characterized using SEM. S.H. conducted ex vivo and in vivo mouse experiments, performed data analysis and drafted the manuscript. J.M. and M.H. designed the experiments, analyzed and interpreted the data and drafted the manuscript. All authors reviewed the manuscript.

\section{Competing interests}

The authors declare no competing interests. 


\section{Additional information}

Supplementary Information The online version contains supplementary material available at https://doi.org/ 10.1038/s41598-021-00765-X.

Correspondence and requests for materials should be addressed to J.L.M.

Reprints and permissions information is available at www.nature.com/reprints.

Publisher's note Springer Nature remains neutral with regard to jurisdictional claims in published maps and institutional affiliations.

(c) (i) Open Access This article is licensed under a Creative Commons Attribution 4.0 International License, which permits use, sharing, adaptation, distribution and reproduction in any medium or format, as long as you give appropriate credit to the original author(s) and the source, provide a link to the Creative Commons licence, and indicate if changes were made. The images or other third party material in this article are included in the article's Creative Commons licence, unless indicated otherwise in a credit line to the material. If material is not included in the article's Creative Commons licence and your intended use is not permitted by statutory regulation or exceeds the permitted use, you will need to obtain permission directly from the copyright holder. To view a copy of this licence, visit http://creativecommons.org/licenses/by/4.0/.

(C) The Author(s) 2021 